\title{
Vitamin E concentration in blood plasma of goats fed palm oil leaves
}

\author{
AR Alimon, B Nor Azam \\ Department of Animal Science, Universiti Pertanian Malaysia, 43400 Serdang Selangor, Malaysia
}

Oil palm is an important crop grown in Malaysia. The pruned oil palm leaf or frond (OPF) is commonly fed as a roughage to ruminants. It has a low crude protein content (4-8\%) and an estimated Metabolizable Energy (ME) of $4.5-5.5 \mathrm{MJ} / \mathrm{kg}$. OPF is also known to contain substantial amounts of vitamin E. Studies by Gapor (1988, JOCS World Congress, 1323-1328) indicated that palm leaves contain $0.14-0.28 \%$ vitamin $E$ mainly in the form of (alpha)-tocopherol.

An experiment was carried out to examine the effect of feeding OPF on the level of vitamin $E$ in blood plasma of goats. Nine male Kacang goats weighing $17-18 \mathrm{~kg}$ were placed in individual pens and given either one of the following treatments ; Diet $A$, control (urea treated rice straw $+200 \mathrm{~g}$ concentrate) ; Diet $\mathrm{B}, \mathrm{OPF}+200 \mathrm{~g}$ concentrate ; Diet $\mathrm{C}$, (urea treated rice straw + concentrate $+60 \mathrm{mg}$ Vitamin $E$ daily). The concentrate contained $75 \%$ corn and $25 \%$ soya-bean meal. The vitamin $E$ was a commercial preparation commonly added to ruminant rations and was given orally together with the concentrate. Chemical analysis showed that diets contained $3.0,410$ and $61 \mathrm{mg}$ vitamin E per $\mathrm{kg}$ dry matter, respectively. The animals were fed the diets for a period of 30 days. Blood samples were taken from the jugular vein at the

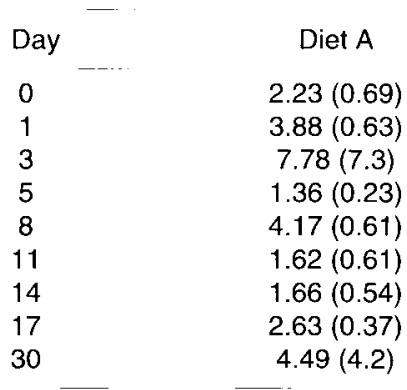

commencement of the trial and thereafter every 3 days. Vitamin $E$ in blood plasma and other blood parameters were also determined. Different diets had little or no effect on blood constituents, such as haemoglobin, plasma protein, PCV, MCV, WBC etc... Plasma vitamin E level (ug/ml) (Table) of goats fed oil palm leaves was higher $(P<0.001)$ than those of goats fed Diet A or Diet C. However, plasma Vitamin $E$ levels of goats on Diets $A$ and $C$ were not significantly different but were within the normal range (Ullrey, 1981, J Anim Sci, 53, 1039-1059). Interestingly, animals on Diet A, although receiving a much lower than the required amounts had an almost similar level of plasma vitamin $E$ as those fed Diet $C$ which supplied the required amount of vitamin $E$ (National Research Council, 1987). This suggests that Vitamin $E$ is efficiently absorbed from the gut and not oxidised in the rumen and supports the findings of Hidiroglou, Butler and Ivan, (1990, Int $J$ Vit and Nutr Res, 60, 4, $331-337)$. It is thought that goats are able to maintain the plasma vitamin $E$ even when the intake is low.

It can be concluded that oil palm fronds can be a good source of vitamin $E$ for livestock. Vitamin $E$ from OPF which is mainly in form of (alpha)-tocopherol is efficiently absorbed and maintained high levels in blood plasma.

Diet B
$4.47(2.15)$
$10.52(11.3)$
$11.41(7.0)$
$12.14(8.73)$
$9.75(4.0)$
$10.26(4.87)$
$14.19(1.97)$
$10.55(8.32)$
$13.08(5.3)$

\section{Diet C}

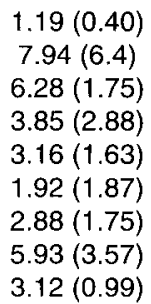

